\title{
SOME SPECIAL VECTOR FIELDS ON A COSYMPLECTIC MANIFOLD ADMITTING A RICCI SOLITON
}

\author{
HALIL İBRAHIM YOLDAŞ, ŞEMSİ EKEN MERIÇ, AND EROL YAŞAR
}

Received 5 February, 2020

\begin{abstract}
In this paper, we deal with the geometric properties of cosymplectic manifold. We give some classifications for a cosymplectic manifold endowed with some special vector fields, such as contact, concircular, recurrent, torse-forming and some characterizations for such a manifold admitting a Ricci soliton given as to be Einstein, $\eta$-Einstein, almost quasi-Einstein and nearly quasi-Einstein.
\end{abstract}

2010 Mathematics Subject Classification: 53C25; 53C40; 53D10

Keywords: Einstein manifold, cosymplectic manifold, Ricci soliton, torse-forming vector field, torqued vector field

\section{INTRODUCTION}

The notion of Ricci soliton, which is a natural generalization of Einstein manifold, was appeared by Hamilton in 1988 [14]. This notion is a fixed point of Hamilton's Ricci flow defined by

$$
\frac{\partial}{\partial t} g=-2 S,
$$

viewed as a dynamical system on the space of Riemannian metrics modulo diffeomorphims and scaling. Also, it models the formation of singularities in the Ricci flow.

In the framework of the contact geometry, Ricci solitons have been studied many mathematicians in some different classes of contact geometry since Sharma applied Ricci solitons to K-contact manifolds [17]. For example, Ghosh proved that if a Kenmotsu 3-manifold is a Ricci soliton then, its constant curvature is -1 in [9]. Turan, De and Yildiz showed that if a 3-dimensional trans-Sasakian manifold with constant scalar curvature admits a gradient Ricci soliton, then it is either a $\beta$-Kenmotsu or an Einstein manifold in [18]. Later, Blaga obtained that there is no Ricci soliton on a Lorentzian para-Sasakian manifold with the potential vector field $\xi$ satisfying conditions $R(\xi, X) . S=0$ and $S . R(\xi, X)=0$ in [1]. For the recent works on Ricci solitons, we refer to $[6,10-12,15,16,19,21,22]$. 
A Riemannian manifold $(M, g)$ is called a Ricci soliton if the following condition is satisfied for arbitrary vector fields $X, Y$ on $M$

$$
\left(f_{V} g\right)(X, Y)+2 S(X, Y)+2 \lambda g(X, Y)=0,
$$

where $£_{V} g$ denotes the Lie-derivative of the metric tensor $g$ along vector field $V, S$ is the Ricci tensor of $M$ and $\lambda$ is a constant. A Ricci soliton is denoted by $(M, g, V, \lambda)$. The vector field $V$ is called the potential vector field of Ricci soliton. If $£_{V} g=0$ and $£_{V} g=\rho g$, then potential vector field $V$ is said to be Killing and conformal Killing, respectively, where $\rho$ is a function. Also, when $V$ is zero or Killing in (1.1), then Ricci soliton reduces to Einstein manifold. Therefore, it is considered as a natural generalization of Einstein metric. In addition, a Ricci soliton is called a gradient if the potential vector field $V$ is the gradient of a potential function $-f$ (i.e., $V=-\nabla f$ ) and is called shrinking, steady or expanding depending on $\lambda<0, \lambda=0$ or $\lambda>0$, respectively.

On the other hand, vector fields have been used for studying diferential geometry of manifolds since they determine most geometric properties of the related object. Also, they have many rich properties and play an important role in the study of Riemannian geometry. That is why, geometric vector fields have been studied extensively by many mathematicians.

Motivated by these circumstances, we study cosymplectic manifolds admitting Ricci solitons with some special geometric vector fields such as concurrent, concircular, recurrent, torse-forming and torqued.

The present paper is organized as follows. In section 2, we give some basic definitions, notations and formulas about almost contact metric manifolds. In section 3, we deal with cosymplectic manifold endowed with some special vector fields and give some relationships between these vector fields. In last section, we investigate cosymplectic manifolds admitting Ricci solitons with concurrent, concircular, recurrent and torse-forming vector field, respectively. Finally, we obtain an important result about cosymplectic manifold which is a gradient Ricci soliton.

\section{PReliminaries}

In this section, we shall review some fundamental definitions and formulas of almost contact metric manifolds from [2], [13] and [20].

A $(2 n+1)$-dimensional differentiable manifold $M$ is called an almost contact metric manifold if there exists an almost contact metric structure $(\varphi, \xi, \eta, g)$ on $M$ and the Riemannian metric $g$ satisfies the following relations:

$$
\varphi^{2} X=-X+\eta(X) \xi, \quad \eta(\xi)=1, \quad \varphi \xi=0, \quad \eta \circ \varphi=0, \quad \eta(X)=g(X, \xi)
$$

and

$$
g(\varphi X, \phi Y)=g(X, Y)-\eta(X) \eta(Y), \quad g(\varphi X, Y)=-g(X, \varphi Y)
$$


for any $X, Y \in \Gamma(T M)$, where $\xi$ is a vector field of type $(0,1)$, (which is so-called the characteristic vector field), 1 -form $\eta$ is the $g$-dual of $\xi$ of type $(1,0)$ and $\varphi$ is a tensor field of type $(1,1)$ on $M$.

On the other hand, in [2], D.E. Blair defined the fundamental 2-form of $M$ as follows:

$$
\Phi(X, Y)=g(X, \varphi Y)
$$

for any $X, Y \in \Gamma(T M)$. Moreover, an almost contact metric structure $(\varphi, \xi, \eta, g)$ on $M$ becomes contact metric structure if the relation

$$
\Phi(X, Y)=d \eta(X, Y)
$$

holds for all $X, Y \in \Gamma(T M)$, where

$$
d \eta(X, Y)=\frac{1}{2}\{X \eta(Y)-Y \eta(X)-\eta([X, Y])\} .
$$

The Nijenhuis tensor field of $\varphi$ is defined by

$$
N_{\varphi}(X, Y)=[\varphi X, \varphi Y]+\varphi^{2}[X, Y]-\varphi[X, \varphi Y]-\varphi[\varphi X, Y]
$$

for all $X, Y \in \Gamma(T M)$. If $M$ is an almost contact metric manifold and the Nijenhuis tensor of $\varphi$ satisfies

$$
N_{\varphi}+2 d \eta \otimes \xi=0
$$

then, $M$ is called a normal contact metric manifold. A normal contact metric manifold $(M, \varphi, \xi, \eta, g)$ is said to be cosymplectic if the following relations hold:

$$
d \eta=0, \quad d \Phi=0 .
$$

Equivalently,

$$
\left(\nabla_{X} \varphi\right) Y=0
$$

for any $X, Y \in \Gamma(T M)$, where $\nabla$ is the Levi-Civita connection on $M$. For a cosymplectic manifold, we also have

$$
\begin{aligned}
\nabla_{X} \xi & =0, \\
S(X, \xi) & =0,
\end{aligned}
$$

where $S$ stands for the Ricci tensor of $M$.

Now, we recall some definitions from [3], [4], [7], [8] and [11] and as follows:

A Riemannian manifold $(M, g)$ is called almost quasi-Einstein and nearly quasiEinstein manifold, respectively if its Ricci tensor field $S$ satisfies

$$
S=c g+d(\beta \otimes \gamma+\gamma \otimes \beta)
$$

and

$$
S=a g+b E
$$


where $a, b, c, d$ are functions, $\beta, \gamma$ are 1 -forms and $E$ is a non-vanishing symmetric (0,2)-tensor on $M$.

Also, a Riemannian manifold $(M, g)$ is called $\eta$-Einstein if there exists two real constants $a$ and $b$ such that the Ricci tensor field $S$ satisfies

$$
S=a g+b \eta \otimes \eta .
$$

If the constant $b$ is equal to zero, then $M$ becomes Einstein.

On the other side, a vector field $V$ on a contact manifold $M$ is called a contact vector field if it preserves the contact form $\eta$, that is,

$$
£_{V} \eta=\mu \eta
$$

for some smooth function $\mu$ on $M$. If $\mu$ vanishes identically in (2.6), then $V$ is called a strict contact vector field. Also, a vector field $V$ on a Riemannian manifold $M$ is called a $\varphi$-analytic if it satisfies

$$
\left(f_{V} \varphi\right)(X)=0
$$

for any $X \in \Gamma(T M)$.

A vector field $v$ on a Riemannian manifold $(M, g)$ is called torse-forming if it satisfies the following condition

$$
\nabla_{X} v=f X+\phi(X) v
$$

for any $X \in \Gamma(T M)$, where $\nabla$ is the Levi-Civita connection on $M, \phi$ is a 1 -form and $f$ is a smooth function on $M$. If the 1-form $\phi$ vanishes identically in (2.7), the vector field $v$ is called a concircular [3]. If $\phi=0$ and $f=1$ in (2.7), then $v$ is called a concurrent vector field [5]. Also, the vector field $v$ is called a recurrent if it satisfies (2.7) with $f=0$.

A nowhere zero vector field $\mathcal{T}$ on a Riemannian manifold $(M, g)$ is called torqued vector field if it satisfies the following two conditions

$$
\begin{array}{rll}
\nabla_{X} \mathcal{T} & =\rho X+\alpha(X) \mathcal{T} \quad \text { and } & \alpha(\mathcal{T})=0
\end{array}
$$

for any $X \in \Gamma(T M)$, where $\nabla$ is the Levi-Civita connection on $M$. The function $\rho$ is called the torqued function and 1-form $\alpha$ is called the torqued form of $\mathcal{T}$.

\section{Cosymplectic Manifold Endowed with Some Special Vector FIELDS}

In this section, we deal with a cosymplectic manifold endowed with a contact, concircular and recurrent vector field and obtain some relationships.

Now, we begin to this section with the following.

Proposition 1. Let $M$ be a cosymplectic manifold endowed with a concircular vector field $v$. Then, the vector field $v$ is a $\varphi$-analytic on $M$. 
Proof. Since $v$ is a concircular vector field, it follows from the definition of the Lie-derivative and from (2.3) we have

$$
\begin{aligned}
\left(£_{v} \varphi\right)(X) & =£_{v} \varphi(X)-\varphi\left(£_{v} X\right)=[v, \varphi(X)]-\varphi([v, X]) \\
& =\nabla_{v} \varphi(X)-\nabla_{\varphi(X)} v-\varphi\left(\nabla_{v} X-\nabla_{X} v\right)=\left(\nabla_{v} \varphi\right) X-f \varphi(X)+\varphi(f X)=0
\end{aligned}
$$

for any $X \in \Gamma(T M)$, which completes the proof.

Next, we shall give the following theorem.

Theorem 1. Let $M$ be a cosymplectic manifold endowed with a vector field $v$. Then, the followings are equivalent to each other:

(i) The vector field $v$ is concircular.

(ii) The vector field $v$ is contact.

Proof. By definition of the Lie-derivative and from (2.4), (2.7), for any $X \in \Gamma(T M)$ one has

$$
\begin{aligned}
\left(£_{v} \eta\right)(X) & =£_{v} \eta(X)-\eta\left(£_{v} X\right)=v(\eta(X))-\eta([v, X]) \\
& =g\left(\nabla_{v} X, \xi\right)+g\left(X, \nabla_{v} \xi\right)-\eta\left(\nabla_{v} X-\nabla_{X} v\right) \\
& =\eta\left(\nabla_{v} X\right)-\eta\left(\nabla_{v} X\right)+\eta\left(\nabla_{X} v\right)=\eta\left(\nabla_{X} v\right)
\end{aligned}
$$

Let us assume that $v$ is a contact vector field. Then, using linearity of $\eta$ and from (2.6), (3.1) we have

$$
\eta\left(\nabla_{X} v\right)=\eta(f X)
$$

for a smooth function $f$, which implies that $v$ is a concircular vector field.

The converse of the theorem is straightforward.

The next theorem gives the relation between strict contact vector field and concircular vector field.

Theorem 2. Let $M$ be a cosymplectic manifold endowed with a concircular vector field $v$. Then, the vector field $\varphi(v)$ is a strict contact on $M$.

Proof. Since $v$ is a concircular vector field on $M$, using the equalities (2.1), (2.3) and (2.4), we get

$$
\begin{aligned}
\left(£_{\varphi(v)} \eta\right)(X) & =£_{\varphi(v)} \eta(X)-\eta\left(£_{\varphi(v)} X\right)=\varphi(v)(\eta(X))-\eta([\varphi(v), X]) \\
& =g\left(\nabla_{\varphi(v)} X, \xi\right)+g\left(X, \nabla_{\varphi(v)} \xi\right)-\eta\left(\nabla_{\varphi(v)} X-\nabla_{X} \varphi(v)\right) \\
& =\eta\left(\nabla_{\varphi(v)} X\right)-\eta\left(\nabla_{\varphi(v)} X\right)+\eta\left(\nabla_{X} \varphi(v)\right)=\eta\left(\nabla_{X} \varphi(v)\right)=0
\end{aligned}
$$

for any $X \in \Gamma(T M)$, which gives that the vector field $\varphi(v)$ is a strict contact on $M$ and the proof is completed.

Considering the above theorems, we have the following:

Remark 1 . If $M$ is a cosymplectic manifold endowed with a contact vector field $v$, then the vector field $\varphi(v)$ is also strict contact on $M$. 
By applying the same method as given in the proof of Theorem 2, we also have the following result.

Theorem 3. Let $M$ be a cosymplectic manifold. Then, the characteristic vector field $\xi$ is a strict contact on $M$.

Using the relations (2.3), (2.4) and (2.7) we get the following propositions.

Proposition 2. Let $M$ be a cosymplectic manifold endowed with a concircular vector field $v$. Then, we have the followings:

(1) If $f$ vanishes identically, then $\xi$ is also concircular on $M$.

(2) $\varphi(v)$ is never concircular on $M$.

Proposition 3. Let $M$ be a cosymplectic manifold endowed with a recurrent vector field $v$. Then, we have the followings:

(1) The vector field $\varphi(v)$ is also recurrent on $M$.

(2) The vector field $\varphi(v)$ is a strict contact on $M$.

\section{Cosymplectic Manifolds Admitting Ricci Solitons}

In this section, we study a cosymplectic manifold admitting a Ricci soliton endowed with some special vector fields such as concurrent, recurrent, torse-forming and torqued vector field, respectively.

The first result of this section is the following.

Proposition 4. Let $M$ be a cosymplectic manifold admitting a Ricci soliton $(M, g, v, \lambda)$ with the potential vector is a concurrent $v$. Then, the followings are satisfied:

(i) $v$ is a conformal Killing on $M$.

(ii) $M$ is an Einstein.

(iii) The Ricci soliton $(M, g, v, \lambda)$ is shrinking.

Proof. For any vector fields $X, Y \in \Gamma(T M)$, it follows from the definition of the Lie-derivative and using (2.7), we have

$$
\left(f_{v} g\right)(X, Y)=g\left(\nabla_{X} v, Y\right)+g\left(\nabla_{Y} v, X\right)=g(X, Y)+g(Y, X)=2 g(X, Y)
$$

which implies that $v$ is a conformal Killing vector field on $M$.

On the other hand, since $M$ is a Ricci soliton and from (1.1), we get

$$
\left(£_{v} g\right)(X, Y)+2 S(X, Y)+2 \lambda g(X, Y)=0 .
$$

Using the relations (4.1) and (4.2), the Ricci tensor of $M$,

$$
S(X, Y)=-(\lambda+1) g(X, Y)
$$

is found which implies (ii).

Furthermore, if we choose $X=Y=\xi$ in (4.3) and using (2.5), one has

$$
\lambda=-1
$$

which proves (iii). 
By applying the same method as given in the proof of Proposition 4, we also have the following result.

Proposition 5. Let $M$ be a cosymplectic manifold admitting a Ricci soliton $(M, g, \varphi(v), \lambda)$ such that $v$ is a concurrent vector field on $M$. Then, the following properties are satisfied:

(i) The vector field $\varphi(v)$ is Killing on $M$.

(ii) $M$ is an Einstein.

(iii) The Ricci soliton $(M, g, \varphi(v), \lambda)$ is steady.

Proposition 6. If $(M, g, \xi, \lambda)$ is a cosymplectic manifold admitting a Ricci soliton, then we have the followings:

(i) The characteristic vector field $\xi$ is Killing on $M$.

(ii) $M$ is an Einstein.

(iii) The Ricci soliton $(M, g, \xi, \lambda)$ is steady.

The next two theorem presents a characterization for a cosymplectic manifold admitting a Ricci soliton to be an almost quasi-Einstein.

Theorem 4. Let $M$ be a cosymplectic manifold admitting a Ricci soliton. If the potential vector field $v$ is a pointwise collinear with $\xi$, then $(M, g, v, \lambda)$ is an almost quasi-Einstein manifold.

Proof. Since that $(M, g, v, \lambda)$ is a Ricci soliton whose potential vector field $v$ is a pointwise collinear with $\xi$, that is, $v=b \xi$, from (2.1) and (2.4), we get

$$
\begin{aligned}
\left(£_{v} g\right)(X, Y) & =g\left(\nabla_{X} v, Y\right)+g\left(\nabla_{Y} v, X\right)=g(X(b) \xi, Y)+g(Y(b) \xi, X) \\
& =X(b) \eta(Y)+Y(b) \eta(X)=g(\nabla b, X) \eta(Y)+g(\nabla b, Y) \eta(X)
\end{aligned}
$$

for any $X, Y \in \Gamma(T M)$, where $\nabla b$ is the gradient of a smooth fuction $b$ on $M$.

By using (1.1) and (4.4), we have

$$
S(X, Y)=-\lambda g(X, Y)-\frac{1}{2}\{g(\nabla b, X) \eta(Y)+g(\nabla b, Y) \eta(X)\} .
$$

If we denote the the dual 1 -form of $\nabla b$ by $\gamma$, then (4.5) becomes

$$
S=-\lambda g-\frac{1}{2}(\gamma \otimes \eta+\eta \otimes \gamma) .
$$

Hence, $(M, g)$ is an almost quasi-Einstein manifold.

Theorem 5. Let $M$ be a cosymplectic manifold endowed with a recurrent vector field $v$ such that $v$ is a pointwise collinear with $\xi$. If $M$ admits a Ricci soliton, then $M$ is an almost quasi-Einstein manifold.

Proof. Since $v$ is a recurrent vector field on $M$, we have

$$
\nabla_{X} v=\phi(X) v
$$


for any $X \in \Gamma(T M)$, where $\phi$ is a 1-form. Also, since the potential vector field $v$ of Ricci soliton is equal to $b \xi$, if we use (2.1), (2.4) and (4.7) then, we get

$$
X(b)=b \phi(X)
$$

for any smooth function $b$ on $M$.

On the other hand, it follows from the definition of Lie-derivative and from (2.1), (4.8), one has

$$
\left(f_{v} g\right)(X, Y)=b \phi(X) \eta(Y)+b \phi(Y) \eta(X) .
$$

Thus, by combining the equation (1.1) with (4.9) we find

$$
S(X, Y)=-\lambda g(X, Y)-\frac{b}{2}(\phi(X) \eta(Y)+\phi(Y) \eta(X))
$$

equivalently,

$$
S=-\lambda g-\frac{b}{2}(\phi \otimes \eta+\phi \otimes \eta)
$$

which proves that $M$ is an almost quasi-Einstein manifold.

Now, we have an another theorem.

Theorem 6. Let $M$ be a cosymplectic manifold endowed with a concircular vector field $v$ such that $v$ is a pointwise collinear with $\xi$. If $M$ admits a Ricci soliton, then $M$ is an $\eta$-Einstein manifold.

Proof. Since $v$ is a concircular vector field, using (2.4), one has

$$
f X=\nabla_{X} v=\nabla_{X} b \xi=X(b) \xi+b \nabla_{X} \xi=X(b) \xi
$$

for any $X \in \Gamma(T M)$. If we take the inner product of (4.10) with $\xi$, we have

$$
X(b)=f \eta(X)
$$

for a smooth function $b$ on $M$.

On the other hand, from definition of the Lie-derivative and using (2.1), (4.4), (4.10) and (4.11), we get

$$
\left(£_{v} g\right)(X, Y)=2 f \eta(X) \eta(Y) .
$$

Therefore, from (1.1) and (4.12) we find

$$
S(X, Y)=-\lambda g(X, Y)-f \eta(X) \eta(Y)
$$

which shows that $M$ is an $\eta$-Einstein manifold.

Using Theorem 6, we have the following remark:

Remark 2. Let $M$ be a cosymplectic manifold endowed with a concurrent vector field $v$ such that $v$ is a pointwise collinear with $\xi$. If $M$ admits a Ricci soliton, then $M$ is an $\eta$-Einstein manifold. 
Theorem 7. Let $M$ be a cosymplectic manifold endowed with a torse-forming vector field $v$ such that $v$ is a pointwise collinear with $\xi$. If $M$ admits a Ricci soliton, then $M$ is a nearly quasi-Einstein manifold.

Proof. Assume that $(M, g, v, \lambda)$ is a Ricci soliton whose potential vector field $v$ is a pointwise collinear with $\xi$. Then, we have

$$
\nabla_{X} v=X(b) \xi
$$

for any $X \in \Gamma(T M)$. Because $v$ is a torse-forming vector field on $M$, using (2.7) and (4.13), we get

$$
X(b)=f \eta(X)+b \phi(X)
$$

where $\phi$ is a 1 -form and $b$ and $f$ are smooth functions on $M$. Also, from (2.1), (4.13) and (4.14), we obtain

$$
\left(£_{v} g\right)(X, Y)=2 f \eta(X) \eta(Y)+b \phi(Y) \eta(X)+b \phi(Y) \eta(X) .
$$

So, if we combine (1.1) with (4.15) we find

$$
S(X, Y)=-\lambda g(X, Y)-f \eta(X) \eta(Y)-\frac{b}{2}(\phi(X) \eta(Y)+\phi(Y) \eta(X)) .
$$

If we take a non-vanishing symmetric $(0,2)$-tensor $E$ in (4.16) such that

$$
E(X, Y)=-f \eta(X) \eta(Y)-\frac{b}{2}(\phi(X) \eta(Y)+\phi(Y) \eta(X))
$$

then, the equation (4.16) reduces to

$$
S=-\lambda g+E .
$$

which gives that $M$ is a nearly quasi-Einstein manifold. Therefore, the proof is completed.

Now, we are ready to give our main theorem.

Theorem 8. Let $M$ be a cosymplectic manifold admitting a Ricci soliton with torqued vector field $\mathcal{T}$ such that $\mathcal{T}$ is a pointwise collinear with $\xi$. Then, either $\mathcal{T}$ is a Killing vector field on $M$ and $M$ is an $\eta$-Einstein manifold or $\mathcal{T}$ is a concircular vector field on $M$ and the Ricci soliton is steady.

Proof. Let $\mathcal{T}$ be a pointwise collinear with the structure vector field $\xi$. That is, $\mathcal{T}=b \xi$, where $b$ is a smooth function on $M$. Then, from (2.4) and (2.8) and from the linearity of the torqued form $\alpha$ we find

$$
\begin{aligned}
X(b) \xi & =\rho X+b \alpha(X) \xi, \\
b \alpha(\xi) & =0
\end{aligned}
$$

for any $X \in \Gamma(T M)$. Taking the inner product of (4.17) with $\xi$, we have

$$
X(b)=\rho \eta(X)+b \alpha(X)
$$


Also, putting $X=\xi$ in (4.19) and using (4.18) gives

$$
\xi(b)=\rho .
$$

Since $M$ is a Ricci soliton with torqued vector field $\mathcal{T}$, from (1.1) and (4.17) one has

$$
X(b) \eta(Y)+Y(b) \eta(X)+2 S(X, Y)+2 \lambda g(X, Y)=0 .
$$

By setting $Y=\xi$ in (4.21) and using (2.5), (4.20) implies

$$
X(b)+\rho \eta(X)+2 \lambda \eta(X)=0 .
$$

Again, setting $X=\xi$ in (4.22) and from (4.20) we derive

$$
\rho+\lambda=0,
$$

From (4.22) and (4.23), we write

$$
X(b)=\rho \eta(X) .
$$

Combining (4.19) with (4.24) yields

$$
b \alpha(X)=0 .
$$

This means that either $b=0$ or $\alpha(X)=0$. If $\alpha(X)=0$, from (2.8) we get that $\mathcal{T}$ is a concircular vector field on $M$. Using the fact that and from (4.21) we find

$$
S(X, Y)=-\lambda g(X, Y)-\rho \eta(X) \eta(Y),
$$

which implies that $M$ is an $\eta$-Einstein manifold.

If $b=0$, from (4.24) we have $\rho=0$. As a result of this, we get $\lambda=0$. Therefore, we get the requested result.

The next theorem gives us a characterization for a cosymplectic manifold endowed with a concurrent vector field $v$ to be a gradient Ricci soliton.

Theorem 9. Let $M$ be a cosymplectic manifold endowed with a concurrent vector field $v$. Then, the characteristic vector field $\xi$ is the gradient of a smooth function $f$ on $M$.

Proof. For any $X \in \Gamma(T M)$ and concurrent vector field $v$, we have

$$
g(v, \xi)=f,
$$

where $f$ is a smooth function on $M$. If we differentiate (4.25) covariantly along arbitrary vector field $X$ and use (2.4) and (2.7) then, we get

$$
g(\nabla f, X)=X g(v, \xi)=g\left(\nabla_{X} v, \xi\right)+g\left(v, \nabla_{X} \xi\right)=g(X, \xi) .
$$

Since the Riemannian metric $g$ is non-degenere, we have

$$
\nabla f=\xi \text {. }
$$

This is the desired result.

As an immediate consequence of Theorem 9, we have the following. 
Corollary 1. Every Ricci soliton $(M, g, \xi, \lambda)$ on a cosymplectic manifold endowed with a concurrent vector field $v$ is gradient.

\section{CONCLUSIONS}

Ricci soliton is a natural generalization of Einstein manifold. This notion corresponds to the self-similar solution of Hamilton's Ricci flow. Over the last decades, the geometry of Ricci solitons has been studied intensively and extensively by many mathematicians on many context. In this paper, we study Ricci solitons on a cosymplectic manifold endowed with some geometric vector fields and give some relationships between these vector fields. Also, we give some necessary conditions for a Ricci soliton to be steady, shrinking. Finally, we give some important characterizations which classifies cosymleptic manifold.

\section{REFERENCES}

[1] A. M. Blaga, " $\eta$-Ricci solitons on Lorentzian para-Sasakian manifolds." Filomat., vol. 30, no. 2, pp. 489-496, 2016, doi: 10.2298/FIL1602489B.

[2] D. E. Blair, Contact manifolds in Riemannian geometry., ser. Lecture Notes in Mathematics, Vol. 509. Springer-Verlag, Berlin-New York, 1976.

[3] B.-Y. Chen, "Some results on concircular vector fields and their applications to Ricci solitons." Bull. Korean Math. Soc., vol. 52, no. 5, pp. 1535-1547, 2015, doi: 10.4134/BKMS.2015.52.5.1535.

[4] B.-Y. Chen, "Classification of torqued vector fields and its applications to Ricci solitons." Kragujevac J. Math., vol. 41, no. 2, pp. 239-250, 2017, doi: 10.5937/kgjmath1702239c.

[5] B.-Y. Chen and D. Sharief, "Ricci solitons and concurrent vector fields." Balkan J. Geom. Appl., vol. 20, no. 1, pp. 14-25, 2015.

[6] M. Crasmareanu, "Parallel tensors and Ricci solitons in $N(k)$-quasi Einstein manifolds." Indian J. Pure Appl. Math., vol. 43, no. 4, pp. 359-369, 2012, doi: 10.1007/s13226-012-0022-3.

[7] U. C. De and A. K. Gazi, "On nearly quasi Einstein manifolds." Novi Sad J. Math., vol. 38, no. 2, pp. 115-121, 2008 .

[8] S. Deshmukh and F. Al-Solamy, "A note on conformal vector fields on a Riemannian manifold." Colloq. Math., vol. 136, no. 1, pp. 65-73, 2014, doi: 10.4064/cm136-1-7.

[9] A. Ghosh, "Kenmotsu 3-metric as a Ricci soliton." Chaos Solitons Fractals, vol. 44, no. 8, pp. 647-650, 2011, doi: 10.1016/j.chaos.2011.05.015.

[10] A. Ghosh, "Certain contact metrics as Ricci almost solitons." Results Math., vol. 65, no. 1-2, pp. 81-94, 2014, doi: 10.1007/s00025-013-0331-9.

[11] A. Ghosh and D. S. Patra, "The $k$-almost Ricci solitons and contact geometry." J. Korean Math. Soc., vol. 55, no. 1, pp. 161-174, 2018.

[12] G. Ghosh and P. Majhi, "Certain results on almost Kenmotsu manifolds with conformal Reeb foliation." Commun. Korean Math. Soc., vol. 33, no. 1, pp. 261-272, 2018.

[13] S. I. Goldberg and K. Yano, "Integrability of almost cosymplectic structures." Pacific J. Math., vol. 31, pp. 373-382, 1969 .

[14] R. S. Hamilton, "The Ricci flow on surfaces," in Mathematics and general relativity (Santa Cruz, CA, 1986), ser. Contemp. Math. Amer. Math. Soc., Providence, RI, 1988, vol. 71, pp. 237-262, doi: $10.1090 /$ conm/071/954419. 
[15] D. M. Naik and V. Venkatesha, " $\eta$-Ricci solitons and almost $\eta$-Ricci solitons on para-Sasakian manifolds," Int. J. Geom. Methods Mod. Phys., vol. 16, no. 9, pp. 1950 134, 18, 2019, doi: 10.1142/S0219887819501342.

[16] A. Sarkar, A. K. Paul, and R. Mondal, "On $\alpha$-para Kenmotsu 3-manifolds with Ricci solitons." Balkan J. Geom. Appl., vol. 23, no. 1, pp. 100-112, 2018.

[17] R. Sharma, "Certain results on $K$-contact and $(k, \mu)$-contact manifolds." J. Geom., vol. 89, no. 1-2, pp. 138-147, 2008, doi: 10.1007/s00022-008-2004-5.

[18] M. Turan, U. C. De, and Y. Ahmet, "Ricci solitons and gradient Ricci solitons in threedimensional trans-Sasakian manifolds." Filomat, vol. 26, no. 2, pp. 363-370, 2012, doi: 10.2298/FIL1202363T.

[19] Venkatesha and H. A. Kumara, "Ricci soliton and geometrical structure in a perfect fluid spacetime with torse-forming vector field," Afr. Mat., vol. 30, no. 5-6, pp. 725-736, 2019, doi: 10.1007/s13370-019-00679-y.

[20] K. Yano and M. Kon, Structures on manifolds, ser. Series in Pure Mathematics. World Scientific Publishing Co., Singapore, 1984, vol. 3.

[21] H. I. Yoldaş, c. Eken Meriç, and E. Yaşar, "On generic submanifold of Sasakian manifold with concurrent vector field," Commun. Fac. Sci. Univ. Ank. Ser. A1. Math. Stat., vol. 68, no. 2, pp. 1983-1994, 2019.

[22] H. I. Yoldaş, c. E. Meriç, and E. Yaşar, "On submanifolds of Kenmotsu manifold with Torqued vector field," Hacet. J. Math. Stat., vol. 49, no. 2, pp. 843-853, 2020, doi: 10.15672/hujms.479184.

Authors' addresses

Halì İbrahim Yoldaş

(Corresponding author) Mersin University, Faculty of Science and Arts, Department of Mathematics, 33343 Mersin, Turkey

E-mail address: hibrahimyoldas@gmail.com

Şemsí Eken Merìç

Mersin University, Faculty of Science and Arts, Department of Mathematics, 33343 Mersin, Turkey

E-mail address: semsieken@hotmail.com

Erol Yaşar

Mersin University, Faculty of Science and Arts, Department of Mathematics, 33343 Mersin, Turkey

E-mail address: eroly69@gmail.com 